\title{
Fahrverbot oder gemeinnützige Arbeit für Steuersünder?
}

\author{
Von Michael Kubiciel, Köln
}

\section{Einleitung}

Der Justizminister des Landes Nordrhein-Westfalen hat vorgeschlagen, Steuerstraftaten künftig mit der Verhängung eines Fahrverbots zu ahnden. Seinen Porsche sechs Monate stehen lassen zu müssen, schmerze einen Zahnarzt mehr als eine Geldstrafe. ${ }^{1}$ Fahrverbote als Strafe für verkehrsbezogene Straftaten sind dem geltenden Recht zwar nicht fremd (II.), auch liegt die Suche nach Alternativen zur Freiheits- und Geldstrafe im (internationalen) Trend (III.). Doch ist ein Fahrverbot weder eine der Rechtsgemeinschaft angemessen erscheinende Reaktion auf Steuerstraftaten noch eine den Täter überzeugende Ermahnung an steuerrechtliche Pflichten. Hingegen erweist sich die, ebenfalls von Justizminister Kutschaty ${ }^{2}$ vorgeschlagene, Verpflichtung zur Erbringung gemeinnütziger Arbeit als verfassungsrechtlich zulässige und praktisch sinnvolle Ergänzung des gegenwärtigen Strafartensystems.

\section{Gegenwärtig: Fahrverbot als Besserungsmaßregel und Denkzettel}

Das deutsche Strafrecht enthält ein differenziertes System verkehrsspezifischer Sanktionen. So erlaubt $\S 69$ StGB dem Strafrichter den Entzug der Fahrerlaubnis, wenn sich aus der Tat ergibt, dass der Straftäter zum Führen von Kraftfahrzeugen ungeeignet ist. Bei dieser Form der Entziehung der Fahrerlaubnis handelt es sich um eine Maßregel der Besserung, die nicht auf die Tat, sondern in die Zukunft blickt und die Gesellschaft vor einem ungeeigneten Kraftfahrer schützen soll. Daher bedarf es neben einer verkehrsspezifischen Anlasstat einer negativen Prognose über die verkehrsspezifische Eignung. § 44 StGB ermöglicht demgegenüber die
Verhängung eines Fahrverbots auch ohne derartige Negativprognose. Voraussetzung ist lediglich eine Straftat, die im Zusammenhang mit dem Führen eines PKWs begangen wurde. Die Existenz dieser Vorschrift folgt mit einiger Zwangsläufigkeit aus dem Umstand, dass Verwaltungsbehörden neben einem Bußgeld ebenfalls ein bis zu dreimonatiges Fahrverbot für eine im Straßenverkehr begangene Ordnungswidrigkeit verhängen dürfen: Wenn eine bloße Ordnungswidrigkeit ein Fahrverbot nach sich ziehen kann, muss dies erst recht bei einer Verkehrsstraftat möglich sein. Der kriminalpolitische Zweck dieses (ordnungsbehördlichen oder strafrechtlichen) Fahrverbots liegt darin, „den Täter vor dem Rückfall zu warnen und ihm ein Gefühl dafür zu vermitteln, was es bedeutet, vorübergehend ohne Führerschein zu sein". ${ }^{3}$ Auch diese Fahrerlaubnisentziehung ist mithin keine echte Strafe, sondern ein Mittel der Prävention. Folgerichtig meinte das BVerfG in einer Entscheidung aus dem Jahr 1969, das Fahrverbot reagiere nicht primär auf begangenes Unrecht, sondern bezwecke eine spezialpräventive Einwirkung auf "pflichtvergessene Kraftfahrer", die zur Beachtung der Verkehrsregeln angehalten werden sollen. ${ }^{4}$ Es handelt sich um eine "Denkzettelstrafe ${ }^{\prime \prime}$, die auf der dritten Stufe des berühmten spezialpräventiven Sanktionenprogramms Franz von Liszts zu verorten ist. ${ }^{6}$

\section{Diversifizierung der Strafarten als internationaler Trend}

\section{Internationale Entwicklung}

Gegenwärtig wird ein Fahrverbot mithin als Maßregel bzw. Denkzettel bei Verstößen gegen verkehrsrechtliche Pflichten angewandt. Ob es hingegen auch als Reaktionsform bei anderen Formen 
der Kriminalität - etwa Steuer- und Wirtschaftsstraftaten - eingesetzt werden kann, ist eine offene Frage. International lässt sich jedenfalls ein Trend zur Reform des strafrechtlichen Sanktionensystems beobachten. Dieser läuft nicht auf die Abschaffung der Strafe hinaus, sondern führt zu einer Ergänzung des klassischen Repertoires an Strafformen ${ }^{7}$. Freiheits- und Geldstrafe werden durch die strafweise Konfiskation von Gegenständen ${ }^{8}$, die Pflicht zur Leistung gemeinnütziger Arbeit ${ }^{9}$ oder die (berühmt-berüchtigten) Ehrenstrafen ${ }^{10}$ ergänzt. Zu einer Veränderung der straftheoretischen Grundlagen sollen diese neuen Strafformen nicht führen, ${ }^{11}$ d.h. die neuen Strafformen sollen auf denselben Grundlagen ruhen wie die tradierten Strafarten Geld- und Freiheitsstrafe.

\section{Nationale Initiativen}

Ob ein Fahrverbot als eine "echte" Hauptstrafe in Deutschland denkbar ist, wird seit Jahrzehnten kontrovers diskutiert. Auch im Zuge der Verhandlungen zur Bildung einer Großen Koalition wurde die Einführung eines Fahrverbotes als eigenständige Sanktion im Erwachsenen- und Jugendstrafrecht diskutiert. ${ }^{12}$ Zuvor hatten sich der 59. und 64. Deutsche Juristentag mit dem Projekt beschäftigt, letztlich aber gegen die Einführung einer solchen Sanktion ausgesprochen ${ }^{13}$. Es sind im Wesentlichen zwei Gründe, die gegen die „Fahrstrafe" geltend gemacht werden: Zum einen wird auf die Höchstpersönlichkeit der Strafe $\mathrm{e}^{14}$ hingewiesen und die fehlende individuelle Vergleichbarkeit der Bedeutung des Autofahrens betont ${ }^{15}$. Einen Bewohner einer ländlichen Region trifft ein Fahrverbot in der Tat härter als den Einwohner einer Großstadt mit ihrem breiten Angebot an (Verkehrs-)Infrastruktur. Für Berufspendler kann ein mehrmonatiges Fahrverbot gar einem Berufsverbot gleichkommen und damit schlechterdings unverhältnismäßig werden. Zum anderen könne gerade bei einem langen Fahrverbot irgendwann der Punkt erreicht sein, an welchem dem (u. U. berechtigten) Drang, das Auto zu nutzen, nachgegeben wird. Für ein Fahren ohne Fahrerlaubnis droht jedoch $\S 21$ StVG eine wei- tere Strafe an: Aus einer Straftat und einer Strafe werden zwei ${ }^{16}$.

Auch spätere rechtspolitische Initiativen scheiterten - zuletzt ein Gesetzesantrag des Bundesrates aus dem Jahr $2008^{17}$. Dieser sah eine Umrechnung eines Geldstrafentagessatzes in einen Tag Fahrverbot im Verhältnis 1:1 vor. Interessant ist der Gesetzesantrag des Bundesrates auch, weil er das entscheidende Argument enthält, mit dem auch die Verhängung eines Fahrverbots für Steuerstraftäter begründet wird: Das Fahrverbot könne finanziell gut situierte Täter stärker beeindrucken als eine Geldstrafe. Dass sich das Fahrverbot je nach Situation unterschiedlich auswirken könne, lässt der Gesetzesantrag nicht als Argument gegen die "Fahrstrafe" gelten. Dies zu Recht: Denn auch Geld- und Freiheitsstrafen entfalten individuell unterschiedliche Wirkungen, ohne dass dies gegen diese beiden tradierten Strafformen geltend gemacht wird. Dagegen spricht die Abhängigkeit der Strafwirkung von der Person des Delinquenten für eine weitere Diversifizierung der Strafformen. Anders gewendet: Es muss Strafarten geben, die Personen treffen, für die eine Geldstrafe kein Übel darstellt, die Verhängung einer Freiheitsstrafe aber schuldunangemessen wäre.

\section{Gemeinnützige Arbeit anstatt Fahrverbot}

\section{Grundlegende Probleme der "Fahrstrafe"}

Aus hiesiger Sicht sprechen zwei, miteinander verbundene Überlegungen gegen die Einführung eines Fahrverbots als Hauptstrafe. Zum einen ist zu berücksichtigen, dass die Strafe ein kommunikativer Akt ist, daher muss die Zufügung von Strafe verdient erscheinen ${ }^{18}$. Es lässt sich jedoch bezweifeln, dass Delinquent und Gesellschaft ein Fahrverbot als verdiente Reaktion für eine Steuerverkürzung erachten. So zeigen Untersuchungen, dass die Bevölkerung ein Fahrverbot lediglich als schuldangemessene Antwort auf eine verkehrsbezogene Straftat ansieht, nicht jedoch auf einen Fall der Steuerverkürzung oder einer Wirtschaftsstraftat. ${ }^{19}$ Trifft dies aber zu, fragt sich, ob der Betroffene sein Fahrverbot tatsächlich als Erinnerung 
an seine Pflichten begreift, die - wie die Steuerpflicht - keinen Bezug zum Straßenverkehr aufweisen.

\section{Gemeinnützige Arbeit}

Bei aller Kritik an dem konkreten Vorhaben - die grundsätzliche Linie der rechtspolitischen Diskussion, die der Vorschlag aus NRW ausgelöst hat, weist in die richtige Richtung: die Verbreiterung des geltenden Strafartensystems. Weil die Strafe auf einen Sozialschaden reagiert, ist sie eine Institution der Gesellschaft ${ }^{20}$ und damit auch einer sozialethischen "Evolution" unterworfen ${ }^{21}$. Daher ist ein Nachdenken über ein zeitgemäßes Strafartensystem überfällig. Viele andere Länder sind Deutschland in diesem Punkt voraus. So wird in Großbritannien über (eine Verschärfung von) Internetverboten als Strafe nachgedacht. Vor allem aber hat in den USA die beklagenswerte Tendenz zu einer "Overcriminalization" (D. Husak) 22 einen positiven Nebeneffekt: Dort werden seit geraumer Zeit neue Strafformen erprobt, zumal die Geldstrafe in den USA weder von der Bevölkerung noch von Gerichten und Politik als adäquate Alternative zur Freiheitsstrafe erachtet wird, sondern eher als "Tarif" für die Inanspruchnahme einer Sonderfreiheit ${ }^{23}$. Hervorgebracht hat dies nicht nur strafersetzende, mediationsähnliche Restorative-Justice-Verfahren, sondern auch die Pflicht zur gemeinnützigen Arbeit als Strafform ${ }^{24}$. Die gemeinnützige Arbeit dient nicht dazu, dem Opfer der Tat Schadensersatz zu leisten, vielmehr soll eine gegenüber der Gesellschaft bestehende Schuld abgearbeitet werden ${ }^{25}$.

Letztere empfiehlt sich als dritte Strafform neben Geld- und Freiheitsstrafe auch für die Aufnahme in das StGB. Auch dem in Deutschland geltenden Recht ist diese Sanktionsfolge nicht fremd. ${ }^{26}$ Gleichwohl wird der Verpflichtung zur gemeinnützigen Arbeit entgegen gehalten, sie sei „persönlichkeitsnegierend", insofern sie auf eine Besserung des Verurteilten abziele ${ }^{27}$, und verstoße gegen die Menschenwürde ${ }^{28}$, weil sie den Verurteilten zu einem "temporären Sklaven" degradiere $^{29}$. Der zuerst genannte Einwand geht zu weit, weil er sämtliche Maßregeln der Besserung trifft. Der zweite Einwand ist eine Polemik, die nicht am Platze ist. Denn die Verpflichtung zur gemeinnützigen Arbeit lässt sich nicht mit der "Kettenstrafe" oder "Sklavereistrafe" früherer Jahrhunderte vergleichen. ${ }^{30}$ Das zeigt sich bereits daran, dass in den USA bemängelt wird, dem "community service" fehle der tadelnd-missbilligende symbolische Gehalt: „We don't ordinarily condemn individuals who perform services such as these (...). ${ }^{131}$ Vor allem aber schließen die Grundrechte eine strafersetzende Arbeitspflicht nicht aus. Art. 4 Abs. 2 EMRK verbietet zwar die Zwangsarbeit, d. h. jede Art von Arbeit oder Dienstleistung, die von einer Person unter Androhung irgendeiner Strafe verlangt wird und für die sie sich nicht freiwillig zur Verfügung gestellt hat ${ }^{32}$. Ausgenommen werden von Art. 4 Abs. 3 a) 1 EMRK lediglich Arbeiten, die üblicherweise von einer rechtmäßig inhaftierten Person verlangt werden. Explizit gestattet ist damit nur die Arbeit in der Haft, nicht Arbeit anstelle von Haft. Die in Abs. 3 genannten Fälle sind jedoch keine Ausnahmen von einem im Übrigen umfassenden Begriff der Zwangsarbeit. Vielmehr werden "normal-übliche Arbeiten" von Art. 4 Abs. 2 EMRK erst gar nicht erfasst. ${ }^{33}$ Daher stellt es keinen Verstoß gegen Art. 4 Abs. 2 EMRK dar, wenn Personen gemeinnützige Arbeit verrichten müssen, um dem Strafvollzug zu entgehen. ${ }^{34}$ Auch verfassungsrechtliche Bedenken bestehen nicht, hat das BVerfG doch klargestellt, dass § 56b Abs. 2 StGB, der die Erbringung gemeinnütziger Arbeiten als Bewährungsauflage zulässt, nicht gegen Art. 12 Abs. 2, 3 GG verstößt. Die Grundrechte bezweckten den Schutz der Person vor Herabwürdigung, so dass begrenzte Arbeitspflichten, die dem Betroffenen durch einen Richter im Rahmen eines gesetzlich ausgeformten und abgestuften Reaktions- und Sanktionssystems als Folge einer Straftat auferlegt werden, grundsätzlich verfassungsrechtlich zulässig seien ${ }^{35}$.

Die gemeinnützige Arbeit ist aber nicht nur eine verfassungsrechtlich zulässige Sanktionsart, sondern den anderen Sanktionsarten überlegen. Nicht nur ist sie kostenärmer als die Freiheitsstrafe, wie Justizminister Kutschaty betont. ${ }^{36}$ Sie ist vor allem 
spürbar und resozialisierungsfreundlich zugleich. Während nämlich Geld- und Freiheitsstrafe lediglich etwas Nur-Negatives implizieren, weil sie dem Delinquenten lediglich Freiheit (in unterschiedlicher Form) entziehen, bringt die gemeinnützige Arbeit auch etwas Positives hervor - für die Gesellschaft und, so ist zu hoffen, auch für den Täter.

\section{Anmerkungen}

1 FAZ v. 25.6.2014.

2 Wie Fn. 1.

3 BT-Drs. IV/651, S. 12.

4 BVerfG NJW 1969, 1623 f. (1624).

5 Zopfs, Festschrift für Wolter, 2013, S. 815, $818 \mathrm{f}$.

6 V. Liszt, ZStW 3 (1883) 1, $41 \mathrm{f}$.

7 Garvey, in: Deigh/Dolinko (Hrsg.), The Oxford Handbook of Philosophy of Criminal Law, 2011, S. $493 \mathrm{ff}$.

8 Mit Verweis auf Österreich Hochmayr, ZStW 124 (2012), 64, $69 \mathrm{ff}$.

9 Für die USA s. Kahan, University of Chicago L. Review 63 (1996), S. 591, 625 ff. Für skandinavische Länder Schneider, in: ders. (Hrsg.), Internationales Handbuch der Kriminologie, Bd. 2, 2009, S. 28 f.

10 Kubiciel, ZStW 118 (2006), S. 44 ff.

11 Gross, Crime and Punishment. A Concise Moral Critique, 2012, S. 172 f. Am Beispiel der Restorative-Justice-Konzeption Garvey (Fn. 7), S. 509 f.

12 Deutschlands Zukunft gestalten, Koalitionsvertrag zwischen CDU, CSU und SPD, 18. Legislaturperiode, S. 146. - Dazu Kubiciel, LTO v. 22.10.2013; Zopfs, NJW 2013, Editorial Heft 52.

13 Verhandlungen des 64. Deutschen Juristentages, 2002, Bd. II/1; Bd. II/2 jew. Teil N; Verhandlungen des 59. Deutschen Juristentages, 1992, Bd. 1 Teil C; Band 2 Teil O.
14 Meier, Strafrechtliche Sanktionen, 3. Aufl. 2009, S. 394.

15 S. auch Zopfs, FS Wolter, 2013, S. 815, 824. Anders König in: Hentschel/König/Dauer, Straßenverkehrsrecht, 42. Auflage 2013, § 21 Rn. 9.

16 Streng ZRP 2004, 237 ff., 240; ders., ZStW 111 (1999), 851 ff.; krit. ferner Weßlau, StV 1999, 285 f.

17 BT Drs. 16/8695.

18 NK-Hassemer/Neumann, StGB, Bd. 1, 4. Aufl. 2013 Vor $\S 1$ Rn. 296.

19 Streng, ZRP 2004, 237, 239.

20 Hassemer/Neumann (Fn. 18), Vor § 1 Rn. 103.

21 Gross (Fn. 11), S. 172.

22 Husak, Overcriminalization 2008.

23 Ausführlich dazu Kahan (Fn. 9), S. 620 ff.

24 Dazu Kahan (Fn. 9), S. 625 ff.

25 S. Sayre-McCord, Philosophical Issue 11 (2001), S. $502,504 \mathrm{f}$.

26 Vgl. § 15 Abs. 1 S. 1 Nr. 3 JGG, Art. 293 EGStGB.

27 Köhler, GA 1987, 145, 156 ff.

28 Roxin, GS Zipf, 1999, S. 135, 147.

29 Köhler, GA 1987, 145, 160.

30 Dazu Koch, in:ders./Kubiciel/Löhnig/Pawlik(Hrsg.),Feuerbachs Bayerisches Strafgesetzbuch, 2014, S. 39, 64 f.

31 Kahan (Fn. 9), S. 626.

32 Esser, in: Löwe-Rosenberg, StPO, Bd. 11, 26. Aufl. 2012, Art. 4 EMRK Rn. 19; Frohwein, in: ders./Peukert, Europäische Menschenrechtskonvention, 3. Aufl. 2009, Art. 4 Rn. 6; Mayer-Ladewig, EMRK, 3. Aufl. 2011, Art. 4 Rn. 5.

33 So Esser (Fn. 32), Art. 4 Rn. 24. S. auch EKMR v. 17.12.1963, Nr. 1468/62 (Iversen) sowie dazu Behnsen, in: Karpensteiner/Mayer (Hrsg.), EMRK, 2012, Art. 4 Rn. 16.

34 Esser (Fn. 32), Art. 4 Rn. 32.

35 BVerfG NJW 1991, 1043, 1044. S. auch BVerfGE 74, $102,120 \mathrm{ff}$.

36 Wie Fn. 1. 